\title{
On Group Communication Systems: Insight, a Primer and a Snapshot
}

\author{
P. Gray $\dagger^{1}$ and J. S. Pascoeł \\ $†$ Math \& Computer Science \\ University of Norther Iowa \\ Cedar Falls, Iowa \\ 50614-0506 \\ gray@math-cs.cns.uni .edu \\ ‡Department of Computer Science \\ University of Reading \\ Reading, UK \\ RG6 6AY \\ J.S.Pascoe@reading.ac.uk
}

\begin{abstract}
This paper contributes a concise introduction to the field of group communication systems and is structured as three integrated parts. In the first instance, this paper aims to share the practical insight gained from the implementation of several group communication projects back into the community. This is discussed in a form that can be used to guide and steer subsequent projects. Secondly, the paper aims to benefit newcomers to the subject by offering an introduction to some of the more pertinent areas of the field through a snapshot of its current state. The subjects of failure detectors, group membership (including virtual synchrony variants) and security are discussed. Although this paper presents a general view on these subjects, it alludes to the exemplars of the Collaborative Computing Frameworks (CCF) and IceT where necessary.
\end{abstract}

Keywords - group communication, state-of-the-art, tutorial, implementation, distributed computing.

\section{Introduction}

The field of group communications is a mature area of distributed computing. Projects such as the CCF [2], IceT [15], Totem [12,3], InterGroup [7], Transis [4] and Horus [17] satisfy the design goals of many applications involving group communications. Numerous presentations of the theoretical work surrounding these projects has been contributed to the community. However, very little of the insight gained from the practical development of this work has been brought together and reintroduced to the field. Thus, this paper aims to present some of this practical insight in a form that can be used to guide future projects. In addition, this article offers a concise primer to the more noteworthy projects and texts that accompany the field.

The pertinent question of which topics to consider is now addressed. A comprehensive consideration of group communication issues would require a much fuller discussion than is given here. In this work, we aim to quickly introduce the reader to, what is in our opinion, the most general and fundamental concepts within the field. Thus, this paper is structured as follows. In section 2 we contrast the use of reliable multicast and keep-alive packets as failure detection mechanisms. Section 3 then discusses the implementation of group membership protocols, virtual synchrony variants and their associated repair algorithms. The issue of security in group communication infrastructures is considered in section 4 and finally, we give our conclusions in section 5 .

\footnotetext{
${ }^{1}$ This research is supported by NSF grant ACI-9872167 and the University of Northern Iowa's Graduate College.
} 


\subsection{Exemplars}

In the interests of generality, the ensuing discussion remains at a high level. However, where necessary, we allude to the exemplar projects of the Collaborative Computing Frameworks (CCF) [2] and IceT [15].

The Collaborative Computing Frameworks The Collaborative Computing Frameworks $(C C F)^{2}$ is a suite of software systems, communications protocols, and methodologies that enable collaborative, computer based cooperative work. The CCF constructs a virtual work environment on multiple computer systems connected over the Internet, to form a collaboratory. In this setting, participants interact with each other, simultaneously access or operate computer applications, refer to global data repositories or archives and conduct a number of other activities via telepresence. CCF is an integrated framework for accomplishing most facets of collaborative work, discussion, or other group activity. It differs from other systems (audio tools, video/document conferencing) in that it aims to provide a comprehensive framework that addresses a broad range of collaborative issues. The CCF software systems are outcomes of ongoing experimental research in distributed computing and collaboration methodologies.

The Collaborative Computing Transport Layer (CCTL) [18] is the fabric upon which the entire system is built. A suite of reliable atomic communication protocols, CCTL supports sessions or heavyweight groups and channels (with relaxed virtual synchrony) that are able to exhibit multiple Qualities of Service (QoS) semantics. This in turn allows applications to operate across differing levels of QoS simultaneously. Unique features include a hierarchical group scheme, use of tunnel-augmented IP multicasting and a multithreaded implementation.

IceT The IceT project aims to provide an environment that is well suited for distributed computing as well as collaborative sessions. Groups of resources are merged together to form metacomputing and collaborative environments. One of the major attributes of the IceT environment is the ability for applications to be 'soft-installed' on demand.

The commonality between distributed and collaborative computing issues in such an environment is the grouping of resources and the hierarchy generated by asymmetric access privileges. That is to say, if two researchers (collaborators) merge their resources together, their local resources typically extend certain privileges to their owners as opposed to remote users. Disk access, CPU usage, etc. are aspects of the environment that might be asymmetrically allocated.

In IceT, access and privileges are based on X509 certificates. Group definitions revolve around access certificates. A group of resources is identified by a common certificate. In the process of merging, where two groups of resources merge together to form a single virtual environment, individual certificates are used to generate common certificates $^{3}$ that represent the combined set of resources. The process of splitting a virtual environment involves heavy use of Certificate Revocation Lists (CRLs). When an environment is split apart, the certificate that identified the virtual composition is revoked, thereby revoking the privileges associated with the alliance.

\footnotetext{
${ }^{2}$ Further information is available at: http: //www. collaborative-computing.com/.

${ }^{3}$ In actuality, a certificate chain is produced that consists of a new certificate and yet retains the qualities of the individual certificates.
} 


\section{Failure Detectors}

A significant portion of recent work has addressed the impossibility of forming consensus on failed processes in asynchronous networks. This result stems from an inherent infeasibility to distinguish between failed processes and those that are arbitrarily slow. Chandra and Toueg [19] proposed eight classes of failure detectors defined in terms of accuracy and completeness. These are summarized in table 1 but for an interesting and more in-depth discussion, the reader is referred to [19].

The implementation of failure detection has often been based on the use of explicit keep-alive packets (or heartbeats). Indeed, Horus [17] and InterGroup [7] both use such a mechanism in their approaches to group communication. Heartbeat packets offer a number of advantages, the most notable being the detection of failed hosts within a known time window. However, a possible drawback of the mechanism is that they impose an unnecessary processing overhead in a failure free system. In wired environments, it is arguable that this overhead is insignificant, but in other networks (e.g. wireless) the processing requirement imposed by heartbeats can use significant levels of resources unnecessarily.

Another approach to failure detection is to augment reliable transmission mechanisms to act as weak failure detectors. Indeed host failures in CCTL are detected through the use of a reliable multicast primitive that has been augmented to provide a weak failure detector. This has the advantage that it does not incur any processing overhead on a failure free system, but a disadvantage of the mechanism is that a failed host can remain undetected for an arbitrary length of time.

The selection of an appropriate failure detector is dependent on the application. For high level collaborative environments such as the CCF, the timely detection of a failed host is often not imperative. Due to this, a failure detector based on a reliable transport mechanism may be adopted. However, in other group communication systems (e.g. safety-critical or process control systems), the detection of a node failure within a specific time period can be crucial to the provision of the service offered [16]. Thus, in this case, a mechanism based on heartbeats is usually more appropriate.

\section{Group Membership}

Group membership protocols are fundamental to any system consisting of a group of communicating processes. Due to network conditions, not all processes are able to communicate with others at all times. Processes can suffer crash failures or the network may partition. Thus, a process can not guarantee that the messages it sends are received by all members of the receiver set. This presents a new collection of failure scenarios that are challenging to deal with.

\begin{tabular}{||c|c|c|c|c||}
\hline \multirow{2}{*}{ Completeness } & \multicolumn{4}{|c||}{ Accuracy } \\
\cline { 2 - 5 } & Strong & Weak & Eventual Strong & Eventual Weak \\
\hline Strong & $\begin{array}{c}\text { Perfect } \\
\mathcal{P}\end{array}$ & $\begin{array}{c}\text { Strong } \\
S\end{array}$ & $\begin{array}{c}\text { EventuallyPerfect } \\
\diamond \mathcal{P}\end{array}$ & $\begin{array}{c}\text { EventuallyStrong } \\
\diamond \mathcal{S}\end{array}$ \\
\hline Weak & $Q$ & $\begin{array}{c}\text { Weak } \\
\diamond Q\end{array}$ & $\diamond Q$ & $\begin{array}{c}\text { EventuallyWeak } \\
\diamond \mathcal{W}\end{array}$ \\
\hline
\end{tabular}

Table 1. The Eight Classes of Failure Detector (see [19] for more details). 
Membership protocols usually provide participant processes with the notion of a view, that is, an ordered list of the current members. All of the members in the current view are guaranteed to either accept the same view, or be removed from the session. Messages sent in the current view are delivered to the surviving members of the current view, and messages received in the current view are received by all surviving members in the current view. This is called Virtual Synchrony [9] and was first implemented in the Isis toolkit [8]. Since all members that can communicate see a failure at the same logical time, the resulting fault-tolerance protocols are significantly simplified.

\subsection{Virtual Synchrony Variants}

Virtual Synchrony is best understood as a simulation of fail-stop behavior, that is, members excluded from the session may still be alive. A number of Virtual Synchrony variants have been proposed and we consider two here.

Extended Virtual Synchrony The standard virtual synchrony model is inherently unequipped to deal with partitioning failures i.e. the model is defined in terms of a single system component in which process groups reside. Thus, in a primary partition system, only the fragment that resides in the primary component of the system can survive. The Extended Virtual Synchrony [12,4] model suggests that applications can tolerate partitioning if processes are allowed to continue in non primary partitions. In such systems, any group of processes that can reach consensus on the membership of the partition is permitted to continue. However the remerging of such states is difficult and can not in general be automated.

Relaxed Virtual Synchrony In CCTL, the virtual synchrony algorithm was extended and optimized for a collaborative setting. To enforce virtual synchrony, all processes must receive the same messages in a given view. Relaxed Virtual Synchrony allows the sending and receiving views to differ. For example, in fig. 1 the receiving view of $m$ contains $d$; the sending view does not. To guarantee virtual synchrony, the sender $b$ compares the sending and receiving views for each message sent. Host $b$ retransmits messages to processes in the receiving view but not in the sending view. If process $b$ fails, all session members will eventually receive notification from the failure detector. Since $b$ may have failed during a multicast operation, some channel members may not have received all messages sent by $b$. Before removing $b$, members must decide which messages from $b$ are to be delivered. CCTL uses a flush procedure similar to that of Isis to perform this task. In brief, messages that have been received by all session members are said to be stable. Thus, the flush procedure removes all messages that are not stable. Virtual synchrony and extended virtual synchrony flush at each view change. CCTL only flushes in the event of a failure since relaxed virtual synchrony allows messages to be delivered in any view. Flushing is expensive as it delays view changes and delivery of subsequent messages. Since other systems generate significant numbers of view changes and also delay message transmission until after the flush terminates, relaxed virtual synchrony offers a somewhat more appropriate solution.

\subsection{Membership Repair Algorithms}

Membership Repair Algorithms (MRAs) are executed on the detection of one or more process crashes. As their name suggests, a membership repair algorithm will restore a group communication systems health in the presence of failures. This is often achieved by forming consensus on the failed members and then installing a corrected view. 


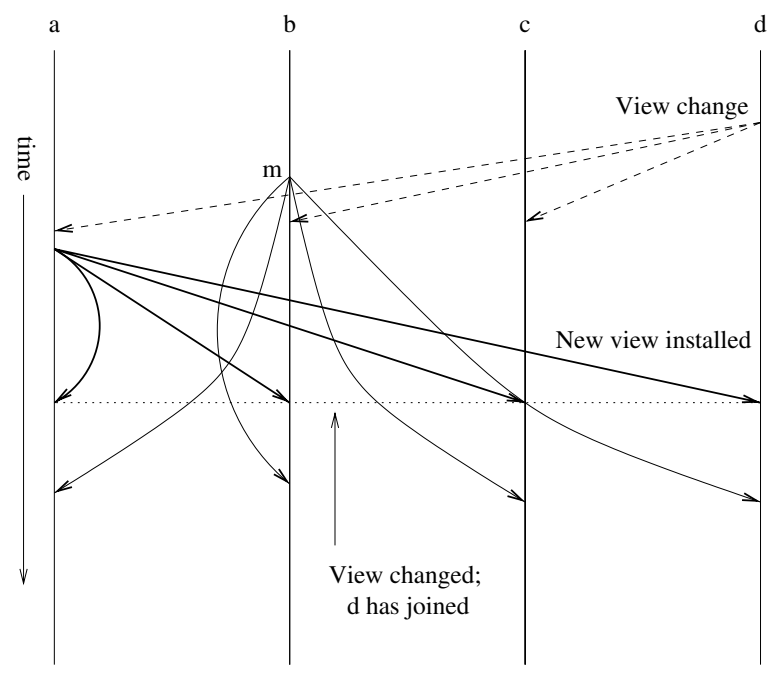

Fig. 1. Relaxed Virtual Synchrony: A view change is required as $d$ makes a request to join the group. Thus, through relaxed Virtual Synchrony, there is no need to flush the system for the outstanding message $m$ since the sending and receiving views are permitted to differ.

A number of novel membership repair algorithms have been suggested. In the CCF, membership repair is achieved through the use of an election based scheme. Each participant in a CCF session executes two threads. The Error Monitor is responsible for the logging of failures and the mediation of the more computationally expensive Error Handler. On detection of a threshold number of consecutive failures, the Error Handler is triggered and a message is multicast to all of the sessions participants informing them that an election is about to take place. Each participant then probes the network to ascertain an up-to-date snapshot of the sessions liveness. These views form the basis of votes which are relayed to the most senior session member (or Error Master) who collates and sorts the votes into a decision on which participants should be removed from the session. This result is multicast to the remaining live session members and the new view is installed.

The Totem Membership Repair Algorithm The Totem MRA is in some respects similar to the strategy employed in the CCF viz. an indication of failure is denoted by a predetermined number of outstanding messages. In Totem, when a failure is detected by a processor, the identification number of the suspect process is added to that processors fail set. The processor then enters the Gather state in which consensus is formed and the corrected view is installed. The algorithm is optimized to yield as large a membership set as possible, whilst not invalidating its termination requirement [1]. For a discussion on additional issues such as token loss and valid membership changes during the Gather state, see [3].

The InterGroup Membership Repair Algorithm InterGroup [7] implements an interesting MRA that is optimized for scalability. The algorithm is divided into two protocols: a lightweight version for passive receivers and a more expensive component that is executed by those processes that are members of the sender set, or wish to become 
members of the sender set in the next view. This approach relies on the assumption that the majority of processors are passive, which given the intended applications of InterGroup, is often the case. Thus, the InterGroup MRA is, in its selected context, an effective method..

\section{Security}

In the context of group-based communication systems that are built upon insecure communication substrates, it is often essential to have security mechanisms in place. This includes addressing aspects of data encryption, mechanisms to authenticate processes and memberships in designated groups, and a way to digitally sign messages.

An associated aspect of security in the context of group-based communication systems is the issue of scalability. The protocol used for scalability should not become an obstacle for 'modest' group memberships. This is especially true in the context of cluster computing and groupware applications, where both the number of interprocess communication channels and the latency of the network are significant security factors.

The topic of security is quite an active research area in general. For a background on general principles and algorithms, see [10], [20], and [5].

\subsection{Key-Pair and Symmetric Ciphers}

One of the fundamental notions to the aspect of security aims at securing private information over a public channel. Briefly, there are two well-known and widely used approaches to this end; key-pair and symmetric ciphers. A cipher is an algorithm that takes the raw form of the information, or plaintext, and transforms it into ciphertext, or information that is unintelligible to an outside party.

A key-pair cipher is an algorithm that is based upon the use of two data sets, or keys. Both keys are required for the process of enciphering and deciphering plaintext. The security of the algorithm hinges upon maintaining close control of one of the keys. This key is known as the private key, and is never disclosed to any outside party. The other key, known as the public key, is freely distributable and open for public inspection. Since both keys are required for encrypting and decrypting information, possession of the public key will not be sufficient enough to compromise the communication process. Given a communication channel between Mary and Bob, Mary can convert plaintext into ciphertext using Bob's public key. This ciphertext would only be able to be deciphered by someone possessing Bob's private key. If Bob's private key is known only to Bob, then Bob would be the only party able to decipher the message. Similarly, Bob could respond to Mary in a secure manner by enciphering plaintext with Mary's public key, etc.

One of the drawbacks of the key-pair approach to encryption is the amount of overhead incurred in the

$$
\text { plaintext } \stackrel{\text { key } 1}{\longrightarrow} \text { ciphertext } \stackrel{\text { key } 2}{\longrightarrow} \text { plaintext }
$$

conversion. A more efficient algorithm is based upon the use of a single session key for both the encipher and decipher processes. That is: 
plaintext $\stackrel{\text { symmetric key }}{\longrightarrow}$ ciphertext $\stackrel{\text { symmetric key }}{\longrightarrow}$ plaintext.

The generation of session keys is often facilitated by an initial key-pair based communication. Examples of these ciphering approaches are given below.

\subsection{Certificate Based Security}

Certificate-based systems rely on Certificate Authorities (CA) and trusted intermediaries to establish authentication of both clients and servers. In a certificate-based system, key-pairs are used to encrypt and decipher messages, to digitally sign message content, and for authentication. Key holders, clients and servers for example, are held responsible for keeping their secret key private. That is, from being compromised. The other key, the public key, is freely open to public inspection. These keys work together for encrypting and decrypting messages. Which key is used for encryption or decryption depends upon the final recipient of the message. Typically, a message originating from a key holder would be encrypted with the private key. The recipient would decrypt the contents with the key holders public key complement.

In a certificate based system, a Certificate Authority (CA) is presented with a key holder's public key and would issue a relatively long-lived credential known as a public key certificate. When two key holder's possess such certificates, they can authenticate each other without further reference to a CA. However, due to the long-lived credential duration, the CA's Certificate Revocation Lists (CRL's) play a role to update revoked credentials. In terms of scalability, a certificate-based system would be limited by the frequency with which group members would need to contact the CA's CRL to learn of revoked certificates.

\subsection{Key Distribution Centers}

Assume that security is based upon a single, secret key technology. That is, where two parties hold the same key for use in encryption and decryption of messages. If the group membership grows fairly large, say $n$ then each group member would need to know $n-1$ keys. Further, the addition of a group member would constitute the generation of $n$ keys. The keys would also have to be able to be distributed securely amongst the group members, which soon becomes an overwhelming task except for all but small group memberships.

One way to make things more manageable is to use a single trusted node known to maintain the keys for all the nodes. This node is called the Key Distribution Center, (KDC). Individual channels are secured between group members by first contacting the KDC for a ticket-granting ticket (TGT), which must then be presented to a centralized Ticket Granting Service (TGS) to obtain a session ticket for the connection. Relative to certificates, the TGT's are short lived, and provides the mechanism to guard against revoked credentials.

Kerberos [13] is an example of an authentication scheme implementation that is built upon the KDC paradigm, supporting many network applications and services. The KDC's make key distribution much more convenient, but there are some major disadvantages too. Mainly, the KDC has enough information to masquerade as any of the 

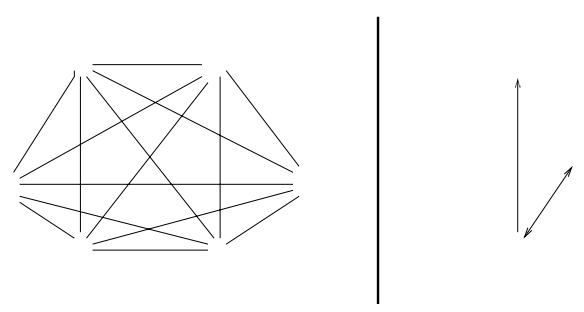

Fig. 2. Using unique session keys for each possible communication channel (left) scales poorly to large groups. Instead, the Ticket Granting Service is used to maintain and distribute session keys on demand (right).

groups members. That is, if it were to be compromised, every connection would be vulnerable. It is a single point of failure in the scenario as well, since if the KDC were to become unreachable, subsequent communication within the group structure would not be possible.

These issues, have to a large extent, been addressed in work such as the Ensemble project. Ensemble has incorporated public key and KDC features into a robust security framework that allows for multiple group partitions, group re-key on demand, and supporting group libraries for building applications that utilize the Ensemble authentication framework. For more information on Ensemble, consult [14].

\section{Conclusion}

This paper has presented tutorial style insight into the central issues surrounding group communication systems. The above discussion is presented in a form that is suitable for new researchers to the field. Where possible, the discussion has attempted to reintroduce some of the implementation insight gained from the development of the wealth of software in the area. In the future, it is envisaged that group communication systems will benefit from the recent advances in the fields of wireless networking and mobile computing. Thus, the consideration of the fields fundamental ideas in these novel contexts poses a series of new and interesting challenges to both the established member of the field, and the newcomer.

\subsection{Recommended Reading}

We briefly draw attention to some of the more noteworthy references in the field. In the authors opinion, the most comprehensive reference on group communication systems is Birman [9]. More detail on Isis, Virtual Synchrony and Hours can be found in numerous papers, available from Cornell's web site ${ }^{4}$. On specifically the concept of Extended Virtual Synchrony, the reader should consult Agarwal's thesis [3]. For security issues, consult the Ensemble project [14]. For synchronous group communication systems, the interested reader is encouraged to study the work of Kopetz [11]. Researchers interested in studying failure detectors should begin by reading the seminal Chandra and Toueg paper [19]. Finally, the work of Holzmann on the SPIN protocol validator [6] provides an interesting starting point for any researchers who are interested in the more formal aspects of the field.

\footnotetext{
${ }^{4}$ See: http://www. cs. cornell. edu/Info/Projects/HORUS/.
} 


\section{References}

1. H. Attiya and J. Welch. Distributed Computing: Fundamentals, Simulations and Advanced Topics. McGraw-Hill, 1998.

2. S. Chodrow, S. Cheung, P. Hutto, A. Krantz, P. Gray, T. Goddard, I. Rhee, and V. Sunderam. CCF: A Collaborative Computing Frameworks. In IEEE Internet Computing, January / February 2000.

3. D. A. Agarwal. Totem: A Reliable Ordered Delivery Protocol for Interconnected Local-Area Networks. PhD thesis, University of California, Santa Barbara, August 1994.

4. D. Dolev and D. Malki. The Transis Approach to High Availability Cluster Communication. In Communications of the ACM, April 1996.

5. Whitfield Diffie and Martin Hellman. New directions in cryptography. IEEE Transactions on Information Theory, IT(22):644-654, November 1976.

6. G. J. Holzmann. Design and Validation of Computer Protocols. Prentice Hall, 1991.

7. K. Berket. The InterGroup Protocols: Scalable Group Communication for the Internet. $\mathrm{PhD}$ thesis, University of California, Santa Barbara, December 2000.

8. K. P. Birman. The Process Group Approach to Reliable Distributed Computing. Communications of The ACM, pages 37-53, December 1993.

9. K. P. Birman. Building Secure and Reliable Network Applications. Prentice Hall, 1997.

10. Charlie Kaufman, Radia Perlman, and Mike Speciner. Network Security: Private communication in a public world. Prentice Hall, Upper Saddle River, New Jersey 07458, 1995.

11. H. Kopetz. Real-Time Systems: Design Principles for Distributed Embedded Applications. Kluwer Academic Publishers, 1997.

12. L. E. Moser and P. M. Melliar-Smith and D. A. Agarwal and R. K. Budhia and C. A. LingleyPapadopoulos. Totem: A Fault-Tolerant Multicast Group Communication System. In Communications of the ACM, April 1996.

13. B. Clifford Neuman and Theodore Y. T'so. Kerberos: An authentication service for computer networks. IEE Communications, pages 33-38, September 94.

14. O. Rodeh and K. P. Birman and D. Dolev. The Architecture and Performance of Security Protocols in the Ensemble Group Communication System. Technical Report TR2000-1791, Cornell University, March 2000.

15. P. Gray and V. S. Sunderam. IceT: Distributed Computing and Java. Journal of Concurrency: Practice and Experience, 9(11):1161-1168, 1997.

16. J. S. Pascoe and R. Loader. A Survey on Safety-Critical Multicast Networking. In Proc. Safecomp 2000, October 2000.

17. R. van Renesse and K. P. Birman and S. Maffeis. Horus, A Flexible Group Communication System. In Communications of the ACM, April 1996.

18. I. Rhee, S. Cheung, P. Hutto, A. Krantz, and V. Sunderam. Group Communication Support for Distributed Collaboration Systems. In Proc. Cluster Computing: Networks, Software Tools and Applications, December 1998.

19. T. D. Chandra and S. Toueg. Unreliable Failure Detectors for Reliable Distributed Systems. Journal of the Association for Computing Machinery, 43(2), 1996.

20. Mary Thompson, William Johnston, Srilekha Mudambai, Gary Hoo, Keith Jackson, and Abdelilah Essiari. Certificate-based access control for widely distributed resources. In Proceedings of the Eighth Usenix Security Symposium, August 99. 